\title{
Reducing the risk of post-surgical cancer recurrence: a perioperative anti-inflammatory anti-stress approach
}

\author{
Rita Haldar ${ }^{1} \&$ Shamgar Ben-Eliyahu*,1 \\ ${ }^{1}$ Sagol School of Neuroscience \& School of Psychological Science, Tel-Aviv University, 69978 Tel-Aviv, Israel \\ *Author for correspondence: shamgar@post.tau.ac.il
}
"We believe that surgery often increases post-surgical risk of cancer recurrence through at least two dormancy-related mechanisms that are triggered by neuroendocrine and paracrine stress-inflammatory responses (SIRs) to surgery."

First draft submitted: 16 November 2017; Accepted for publication: 30 November 2017; Published online: 6 April 2018

Keywords: $\beta$-blocker $\bullet$ cancer progression $\bullet$ dormancy $\bullet$ etodolac $\bullet$ inflammation $\bullet$ metastasis $\bullet$ NSAID $\bullet$ perioperative storm $\bullet$ preoperative balance $\bullet$ propranolol $\bullet$ stress $\bullet$ surgery

Cancer is a worldwide health problem, and is the second leading cause of death in the USA [1]. Importantly, metastatic disease remains the major cause of cancer mortality, despite advances in early detection and therapeutic interventions that have improved survival rates in many types of cancer [1]. For example, 5-year survival rates can reach up to $95 \%$ in breast cancer (BC) patients and $90 \%$ in colorectal cancer (CRC) patients when the primary tumor (PT) is detected at a localized stage, but drop to approximately $25 \%$ and $13 \%$, respectively, if the disease has spread to distant organs [1]. Thus, understanding the mechanisms involved in pro-metastatic malignant cell transformations and in metastatic progression bears potential clinical advantages through the development of novel anti-metastatic therapeutic approaches.

Here, we discuss the critical impact of the perioperative period on long-term cancer outcomes. This delicate period can act to destabilize the balance between pro- and anti-metastatic signals, potentially creating a 'snowball effect', ultimately leading to metastatic outbreak. On the other hand, the perioperative period can be favorable as it presents a window of opportunity to reverse the balance toward disease arrest, through promoting a fast-growingto-dormant transformation and/or preventing an escape from cancer dormancy in residual malignant disease. This critical period remains mostly unexploited clinically, and we suggest herein that a perioperative treatment that results in $\beta$-adrenergic blockade and COX2 inhibition will tip the scale toward disease arrest, as indeed suggested by two Phase II biomarker clinical trials we recently conducted, and by clinical observational studies.

\section{Non-proportional high impact for the short perioperative time-frame on metastatic progression \& outbreak}

Surgery for the removal of a PT is a life-saving procedure in most cancer types. However, the short perioperative period, defined as the days before to weeks post tumor excision, was suggested to facilitate the formation of new metastases and to promote the growth or outbreak of preexisting micrometastases, through numerous prometastatic, pro-angiogenic and immune-suppressive mechanisms [2]. On the other hand, surgical removal of the PT was also suggested to prevent or regress metastatic progression through various mechanisms. These include: cessation of tumor cells shedding into the circulation and lymphatic system, elimination of pro-metastatic factors secreted by the PT (e.g., IL-6, IL-8, VEGF), and the termination of immune-suppressive effects of the excised PT. These multiple pro- and anti-metastatic processes occur simultaneously during the short perioperative period, and their cumulative impact can tip the scale toward metastatic growth, or alternatively, toward disease arrest or elimination.

We believe that before surgery, in patients without evident metastatic disease, a dynamic balance exists between pro- and anti-metastatic processes (i.e., 'the pre-operative balance'), with a possible slight inclination toward

Future 8 Medicine 
metastatic progression. However, on many occasions, the perioperative storm of pro- and anti-metastatic effects (detailed in [3]) tips the scale toward metastatic outbreak. Moreover, pro-metastatic processes synergistically activate each other, creating a self-perpetuating 'snowball effect', resulting in disease recurrence. Nevertheless, perioperative therapeutic interventions targeting one or more of the pro-metastatic processes can potentially tip the scale toward disease arrest or elimination. Thus, the perioperative period is critical in determining the risk for post-operative metastatic disease, and offers a window of therapeutic opportunity against residual malignant disease. Unfortunately, this window of opportunity remains mostly unexploited clinically.

Stress \& surgery may promote post-surgical metastatic outbreak, through prevention of fast-growing-to-dormant transformation \& through induction of an escape from dormancy

A critical aspect in post-operative metastatic disease is the potential perioperative existence of micrometastases, which are either in a dormant or growing state. Dormant metastases are usually small sized, avascular and asymptomatic, and can remain dormant for prolonged periods (e.g., [4]). There is an ongoing debate regarding the nature and definition of cancer dormancy, and several mechanisms have been suggested (reviewed in [5]), including: malignant cell quiescence, balance between proliferation and apoptosis within malignant foci, partial immune control over residual malignant disease and/or angiogenic dormancy, where the lack of malignant tissue vascularization limits its growth [5]. Similar to the 'pre-operative balance', these mechanisms often involve equilibrium between promotion and arrest of malignant growth. Through yet unknown triggers, this equilibrium can be destabilized, causing latent micrometastases to escape from dormancy, thus yielding fast-growing metastatic foci and disease recurrence.

We believe that surgery often increases post-surgical risk of cancer recurrence through at least two dormancyrelated mechanisms that are triggered by neuroendocrine and paracrine stress-inflammatory responses (SIRs) to surgery. First, SIRs may prevent potential fast-growing-to-dormant transformation of growing micrometastases. Without SIRs, these growing metastases may become dormant, given the elimination of metastatic-supporting signals from the removed PT, as detailed below. Second, SIRs may promote an escape from dormancy of established dormant micrometastases. For example, stress and inflammatory responses are known to elevate VEGF secretion, thus eliciting an escape from angiogenic dormancy, and cause immune suppression, thereby jeopardizing immune control over metastatic progression.

Unfortunately, the lack of established experimental models of post-operative spontaneous dormant metastases, and the limited clinical accessibility to dormant metastases, are two major obstacles for studying the above assertions. During the last 2 years, employing advanced imaging techniques, we have been developing two orthotropic models of spontaneous metastases in which, following removal of the PT with minimal surgical procedure, micrometastases have transformed into a latent, non-progressing state for at least 50 days. These models are based on a human breast cancer cell-line (MDA-MB-231 ${ }^{\mathrm{HM}}$ ) in nude mice [6], and on a syngeneic melanoma (B16F10) in C57BL/6J mice (Unpublished Data). Such models can enable the critical exploration of molecular, cellular, and systemic mediating mechanisms underlying both fast-growing-to-dormant transformation and escape from dormancy of metastatic foci, as well as the role of SIRs in these processes.

Indeed, using the MDA-MB- $231^{\mathrm{HM}}$ model, we demonstrated that micrometastases depend on factors secreted by the PT for their continuous growth, and that within the first $24 \mathrm{~h}$ following PT removal, metastases dramatically regress in size and halt their progression thereafter [6]. Implantation of osmotic mini-pumps containing conditioned medium of MDA-MB-231 ${ }^{\mathrm{HM}}$ cells immediately after PT removal diminished the regression of metastases. Furthermore, excision of the PT at a later stage, when the metastases are larger and presumably more self-sufficient, results in lower regression. To identify pivotal PT-secreted factors underlying the pro-metastatic effects of the PT, we conducted proteomic analyses and quantified cytokine levels in the serum of tumor-bearing mice and in conditioned medium, and found significant indications for the involvement of eight cytokines (i.e., IL-6, IL-8, VEGF, EGF, PDGF-aa, MIF, serpinE1 and M-CSF). Furthermore, in vivo simultaneous blockade of IL-8, PDGF-aa, MIF, and serpinE1, without removing the PT, resulted in a partial regression of metastases. Together, these results demonstrate the destabilization of the pre-operative balance, leading to an arrest of micrometastases progression following PT removal. On the other hand, and as detailed below, other destabilizing factors, such as an extensive surgery, can tip the balance toward the opposite direction of metastatic outbreak, even when the PT is removed. Additional studies of the involvement of SIRs in both of these processes are necessary, and are currently being undertaken. 
The role of catecholamines \& prostaglandins in post-surgical metastatic risk

Catecholamines (CAs) and prostaglandins (PGs; specifically PGE2), which are abundant perioperatively, were repeatedly shown to mediate numerous pro-metastatic effects of stress and surgery, through various mechanisms [2,7]. For example, these ligands were shown to regulate the secretion of pro- and anti-inflammatory soluble factors (e.g. IL6, CRP, TNF- $\alpha$ and IL-10), locally and systemically, to suppress NK and T-cell cytotoxicity, thus promoting cancer metastasis [2], and to directly impact tumor cells, promoting their growth, motility, resistance to cell death (apoptosis and anoikis), epithelial-to-mesenchymal transition [8] and secretion of pro-angiogenic and pro-metastatic factors (e.g., VEGF, MMP2, MMP9) [2]. Recently, neuroendocrine stress responses were also suggested to promote an escape from dormancy of cancer cells, as adverse events activate the same molecular pathways suspected to induce an escape from dormancy [9]. The above numerous deleterious effects of CAs and PGs occur simultaneously during the perioperative period, synergistically enhancing their impact in determining the risk of post-operative metastatic disease. Therefore, a perioperative combined blockade of CAs and PGs may prove beneficial.

Indeed, pre-clinical in vitro and in vivo studies have indicated that $\beta$-adrenergic blockade and/or PGs synthesis inhibition can reduce the immune-suppressive and pro-metastatic effects of stress and surgery in several tumor lines and models (e.g., [10-12]). Specifically, the perioperative use of the $\beta$-adrenergic blocker, propranolol and the COX2 inhibitor, etodolac, was shown to be safe at the perioperative setting, and reduced post-operative metastases and mortality rates (reviewed in [2]). Importantly, in some models, only the simultaneous use of the two drugs was effective [12-14], specifically when survival rates were tested following PT excision. As CAs and PGs are both abundantly released perioperatively, and as each can promote metastasis through activating the cAMP-PKA intracellular pathways in immune or malignant cells [2], blocking only one of them may not suffice to improve longterm cancer outcomes. With respect to cancer dormancy, we recently found that the addition of extensive surgery (i.e., laparotomy) to the minimal procedure necessary for PT excision, prevented the fast-growing-to-dormant transformation evident in the MDA-MB- $231^{\mathrm{HM}}$ model, while the combined perioperative use of propranolol and etodolac attenuated the surgery-induced metastatic outbreak [6].

\section{Reducing post-surgical risk for cancer recurrence in cancer patients, employing a perioperative} combined anti-stress-inflammatory treatment, using propranolol \& etodolac

Thus far, the potential long-term clinical impact of $\beta$-blockers and/or COX2 inhibitors in cancer patients has not been clarified (discussed in $[15,16]$ ). However, most studies assessing the impact of such medications were retrospective, rather than randomized-controlled trials, and did not focus on perioperative use of such drugs, but rather on long-term intake initiated before cancer diagnosis. Additionally, concerns were raised regarding the safety of the perioperative use of the $\beta 1$-selective antagonist, metoprolol, in the POISE trial [17], yet no evidence indicates a risk associated with use of non-selective $\beta$-adrenergic antagonists, such as propranolol. Furthermore, propranolol use was associated with reduced post-operative cardiac complications (elaborately discussed in the Supplementary of [18]) and reduced risk of cancer development, especially after prolonged use [19]. Similarly, a recent meta-analysis has identified a protective effect of COX-2 inhibitors against BC [20].

During the last 6 years, we have studied, for the first time in clinical trials, the combined perioperative use of propranolol and etodolac. Two randomized placebo-controlled biomarker clinical trials were conducted in BC and in CRC patients, with treatment beginning 5 days before surgery, assessing pro-inflammatory and pro-metastatic characteristics of the excised malignant tissue and of peripheral bloods.

In both studies, transcriptome profiling of the PT, based on a priori hypotheses, indicated that drug treatment decreased tumor epithelial-to-mesenchymal transition, reduced activity of pro-metastatic and pro-inflammatory transcription factors associated with malignant progression, and decreased tumor-infiltrating monocytes, together suggesting reduced metastatic potential of the malignant tissue [18,21]. In CRC patients, there was an increase in tumor-infiltrating NK cells, which seem to be of special interest, as tumor-infiltrating NK cells were reported to be associated with improved survival [22]. In BC patients, where repeated blood samples were taken, drug treatment also reversed an increase in serum IL- 6 and CRP levels evident in the placebo-control group on the morning before surgery, and prevented a perioperative increase in serum levels of IFN- $\gamma$ and of TRAIL. These outcomes were supported with corresponding effects on both the intracellular mRNA expression levels and the transcriptional activity related to those cytokines [23]. Additionally, drug treatment also blocked a post-operative influx of circulating $\mathrm{CD} 14^{++} \mathrm{CD} 16^{-}$monocytes, and enhanced the expression of the activation marker CD11a (LFA-1) on circulating NK cells [18]. The numerous indices positively affected by the drug treatment in these two Phase II clinical trials 
establish an empirical basis and provide medical justification for large clinical trials to assess the impact of this treatment on disease recurrence and overall survival.

Noteworthy, for patients without contraindications, the safety profiles of propranolol and etodolac are well established, especially for the short duration employed in our clinical studies (elaborately discussed in the Supplementary of [18]). In both of our Phase II clinical studies [18,21], drugs were well tolerated, with minor adverse event rates equivalent to placebo group. To ascertain the long-term safety of the drug regiment, in the CRC clinical trial we also recorded 3-year recurrence rates. In the placebo group, recurrence rates mounted to $29 \%$ (5/17 patients - as would be expected), whereas in the drug-treated group rates reached $7 \%(1 / 15)$, suggesting the long-term safety and potential efficacy of the treatment $(\mathrm{p}=0.154)$ [21]. Thus, any hypothetical long-term risk associated with the combined use of propranolol and etodolac appears unlikely and should be weighed against the positive outcomes reported by translational, epidemiologic and clinical studies such as the above.

\section{Conclusion \& future perspective}

Given pre-clinical and clinical observations described above, we believe that cancer mortality could be reduced by a safe perioperative blockade of SIRs. It is now justified and crucial to conduct larger clinical trials, assessing disease-free survival (DFS) and overall survival (OS), as without these long-term outcomes such interventions will not become a clinical routine. However, recruiting funds for such large trials is an ongoing challenge, as drugs that can be used for this purpose (e.g., propranolol and etodolac) are not patented and are inexpensive, and thus such trials are unattractive for pharmaceutical companies.

Unfortunately, propranolol and/or etodolac are contraindicated for approximately $50 \%$ of patients (mostly due to asthma, cardiovascular disease, diabetes or low blood pressure). Therefore, the development of alternative approaches to limit perioperative SIRs is required. For patients with contraindications to etodolac, alternative antiinflammatory approaches such as statins and omega-3 intake could be considered (reviewed in [2]). For patients with contraindications to propranolol, we have been developing a 6-week perioperative stress-management intervention, which can be employed instead of propranolol, or as a supplementary treatment to the combined drug treatment. This perioperative stress-management intervention aims to address physiological and cognitive aspects of stress responses, as well as resolution of medical uncertainties and management of social support, based on individual patient needs. To the best of our knowledge, such perioperative stress-inflammatory-reducing intervention has not been studied previously, and we believe that together with a perioperative administration of propranolol and etodolac, such a bio-behavioral approach may prove advantageous in reducing post-surgical risk for cancer recurrence.

Future studies of the effects of stress and inflammatory responses on fast-growing-to-dormant transformation, and on escape from dormancy of metastatic foci are needed, as dormancy of residual malignant disease seems to be a major concern in post-surgical risk for cancer recurrence. Such studies should address mechanisms via which stress and/or surgery prevent the induction of dormancy in metastatic foci immediately following PT removal, and/or promotes the outbreak of established dormant micrometastases long following PT excision. Lastly, the effects of pharmacological interventions against SIRs, in reversing such dormancy-related effects, should also be studied, aiming to attenuate metastatic outbreak of dormant malignancies.

Financial \& competing interests disclosure

The authors have no other relevant affiliations or financial involvement with any organization or entity with a financial interest in or financial conflict with the subject matter or materials discussed in the manuscript apart from those disclosed.

No writing assistance was utilized in the production of this manuscript.

Open access

This work is licensed under the Attribution-NonCommercial-NoDerivatives 4.0 Unported License. To view a copy of this license, visit http://creativecommons.org/licenses/by-nc-nd/4.0/

\section{References}

1. Siegel RL, Miller KD, Jemal A. Cancer statistics, 2016. CA Cancer J. Clin. 66(1), 7-30 (2016).

2. Horowitz M, Neeman E, Sharon E, Ben-Eliyahu S. Exploiting the critical perioperative period to improve long-term cancer outcomes. Nat. Rev. Clin. Oncol. 12(4), 213-226 (2015). 
3. Neeman E, Ben-Eliyahu S. Surgery and stress promote cancer metastasis: new outlooks on perioperative mediating mechanisms and immune involvement. Brain Behav. Immun. 30(Suppl.), S32-S40 (2013).

4. Tsao H, Cosimi AB, Sober AJ. Ultra-late recurrence (15 years or longer) of cutaneous melanoma. Cancer 79(12), 2361-2370 (1997).

5. Aguirre-Ghiso JA. Models, mechanisms and clinical evidence for cancer dormancy. Nat. Rev. Cancer 7(11), 834-846 (2007).

6. Shaashua L, Sloan EK, Satchi-Fainaro R, Geiger T, Erez N, Ben-Eliyahu S. Promotion of micrometastases by primary tumor secretome, and the deleterious effects of inflammatory surgical-stress responses on dormant metastases. Presented at: Psychoneuroimmunology Research Society Conference. Brighton, UK, June 2016.

7. Neeman E, Zmora O, Ben-Eliyahu S. A new approach to reducing postsurgical cancer recurrence: perioperative targeting of catecholamines and prostaglandins. Clin. Cancer Res. 18(18), 4895-4902 (2012).

8. Neil JR, Johnson KM, Nemenoff RA, Schiemann WP. Cox-2 inactivates Smad signaling and enhances EMT stimulated by TGF-beta through a PGE2-dependent mechanisms. Carcinogenesis 29(11), 2227-2235 (2008).

9. Zappala G, McDonald PG, Cole SW. Tumor dormancy and the neuroendocrine system: an undisclosed connection? Cancer Metastasis Rev. 32(1-2), 189-200 (2013).

10. Inbar S, Neeman E, Avraham R, Benish M, Rosenne E, Ben-Eliyahu S. Do stress responses promote leukemia progression? An animal study suggesting a role for epinephrine and prostaglandin-E2 through reduced NK activity. PLoS ONE 6(4), e19246 (2011).

11. Le CP, Nowell CJ, Kim-Fuchs $\mathrm{C}$ et al. Chronic stress in mice remodels lymph vasculature to promote tumour cell dissemination. Nat. Commun. 7, 10634 (2016).

12. Sorski L, Melamed R, Matzner P et al. Reducing liver metastases of colon cancer in the context of extensive and minor surgeries through beta-adrenoceptors blockade and COX2 inhibition. Brain Behav. Immun. 58, 91-98 (2016).

13. Benish M, Bartal I, Goldfarb Y et al. Perioperative use of beta-blockers and COX-2 inhibitors may improve immune competence and reduce the risk of tumor metastasis. Ann. Surg. Oncol. 15(7), 2042-2052 (2008).

14. Glasner A, Avraham R, Rosenne E et al. Improving survival rates in two models of spontaneous postoperative metastasis in mice by combined administration of a beta-adrenergic antagonist and a cyclooxygenase-2 inhibitor. J. Immunol. 184(5), 2449-2457 (2010).

15. Fitzgerald PJ. Beta blockers, norepinephrine, and cancer: an epidemiological viewpoint. Clin. Epidemiol. 4, 151-156 (2012).

16. Stasinopoulos I, Shah T, Penet MF, Krishnamachary B, Bhujwalla ZM. COX-2 in cancer: Gordian knot or Achilles heel? Front Pharmacol. 4, 34 (2013).

17. Group PS, Devereaux PJ, Yang H et al. Effects of extended-release metoprolol succinate in patients undergoing non-cardiac surgery (POISE trial): a randomised controlled trial. Lancet 371(9627), 1839-1847 (2008).

18. Shaashua L, Shabat-Simon M, Haldar R et al. Perioperative COX-2 and beta-adrenergic blockade improves metastatic biomarkers in breast cancer patients in a Phase-II randomized trial. Clin. Cancer Res. 23(16), 4651-4661 (2017).

19. Chang PY, Huang WY, Lin CL et al. Propranolol reduces cancer risk: a population-based cohort study. Medicine (Baltimore) 94(27), e1097 (2015).

20. De Pedro M, Baeza S, Escudero MT et al. Effect of COX-2 inhibitors and other non-steroidal inflammatory drugs on breast cancer risk: a meta-analysis. Breast Cancer Res. Treat. 149(2), 525-536 (2015).

21. Haldar R, Ricon I, Cole S, Zmora O, Ben-Eliyahu S. Perioperative beta-adrenergic blockade and COX2 inhibition in colorectal cancer patients improves pro-metastatic indices in the excised tumor: EMT, tumor infiltrating lymphocytes (TILs), and gene regulatory pathways. Presented at: PNIRS 2017 24th Annual Scientific Meeting. Galveston, TX, USA, June 2017.

22. Coca S, Perez-Piqueras J, Martinez D et al. The prognostic significance of intratumoral natural killer cells in patients with colorectal carcinoma. Cancer 79(12), 2320-2328 (1997).

23. Haldar R. Peri-operative blockade of COX2 and $\beta$-adrenoceptors in breast cancer patients: effects on PBMCs transcriptome and serum cytokine levels [Ma thesis]. School of Psychological Sciences, Tel-Aviv University, Tel-Aviv, Israel 1-31 (2017). 
